УДК 629.5.018.26

\title{
РАЗРАБОТКА СТЕНДА ДЛЯ ИССЛЕДОВАНИЯ ФУНКЦИОНИРОВАНИЯ МИКРОМЕХАНИЧЕСКИХ ПРИБОРОВ
}

Кириленко Ярослав Владимирович

студент

Сакович Сергей Юрьевич

к.т.н., доцент

ФГБОУ ВО «Санкт-Петербургский государственный морской технический университет»

\begin{abstract}
Аннотация: Данная статья посвящена разработке интерактивного обучающего стенда, предназначенного для исследования работы блока микромеханических датчиков, гироскопа и акселерометра. В статье рассматривается структура стенда, электрическая схема, протокол взаимодействия с управляющим компьютером и разработанное программное обеспечение для отображения измерительной информации.
\end{abstract}

Ключевые слова: микроконтроллер, гироскоп, акселерометр, микромеханические датчики, лабораторный стенд.

\section{DEVELOPMENT OF A STAND FOR THE STUDY OF THE FUNCTIONING OF MICROMECHANICAL DEVICES}

\section{Kirilenko Yaroslav Vladimirovich Sakovich Sergey Yurevich}

\footnotetext{
Abstract: This article is devoted to the development of an interactive training stand designed to study the operation of a block of micromechanical sensors, a gyroscope and an accelerometer. The article discusses the structure of the stand, the electrical circuit, the protocol of interaction with the control computer and the developed software for displaying measurement information.

Key words: microcontroller, gyroscope, accelerometer, micromechanical sensors, stand.
} 
Широкое использование автоматизации в различных технических средствах, используемой как в повседневной, так и хозяйственной деятельности человека, привело к активному развитию электронной компонентной базы, а также необходимости подготовки специалистов для разработки новых устройств и обслуживания существующих систем. Расширение электронной элементной базы, совместно с применением модульности и унификации, привело к появлению функциональных блоков, объединяющих периферийное и интерфейсное обеспечение. На данный момент, существует большое количество модулей электронных устройств различных по назначению, среди которых можно выделить блоки микромеханических датчиков, обеспечивающих получение данных об ориентации объекта и его перемещении. Подобные блоки используются как элементы обратной связи в системах автоматического управления роботов и аппаратов различного назначения. Исследование работы микромеханических датчиков, а также организации подготовки студентов по использованию и обслуживанию таких датчиков, потребовало разработки лабораторного стенда.

На начальном этапе разработки был проведен анализ существующих микромеханических приборов, которые могли бы выполнять работу гироскопа и акселерометра, имитируя действие морских навигационных приборов. Изучив возможные варианты, был выбран модуль GY-521, который позволяет выполнять указанные задачи, и имеет совместимость с микроконтроллерами по интерфейсу I2C. Для управления модулем датчиков и получения измеренных данных использован микроконтроллер ATmega328, на базе отладочной платы ArduinoUNO. Применение унифицированных модулей функциональных устройств и отладочных наборов ускоряет процесс разработки и упрощает отладку. Вместе с этим, такой подход обеспечивает высокую ремонтопригодность, масштабируемость стенда и защиту пользователей при работе.

Функциональный модуль GY-521 построен на микросхеме серии MPU-6050[1]. На основе анализа технической документации, была разработана схема подключения блока GY-521 к микроконтроллеру (рис. 1). 


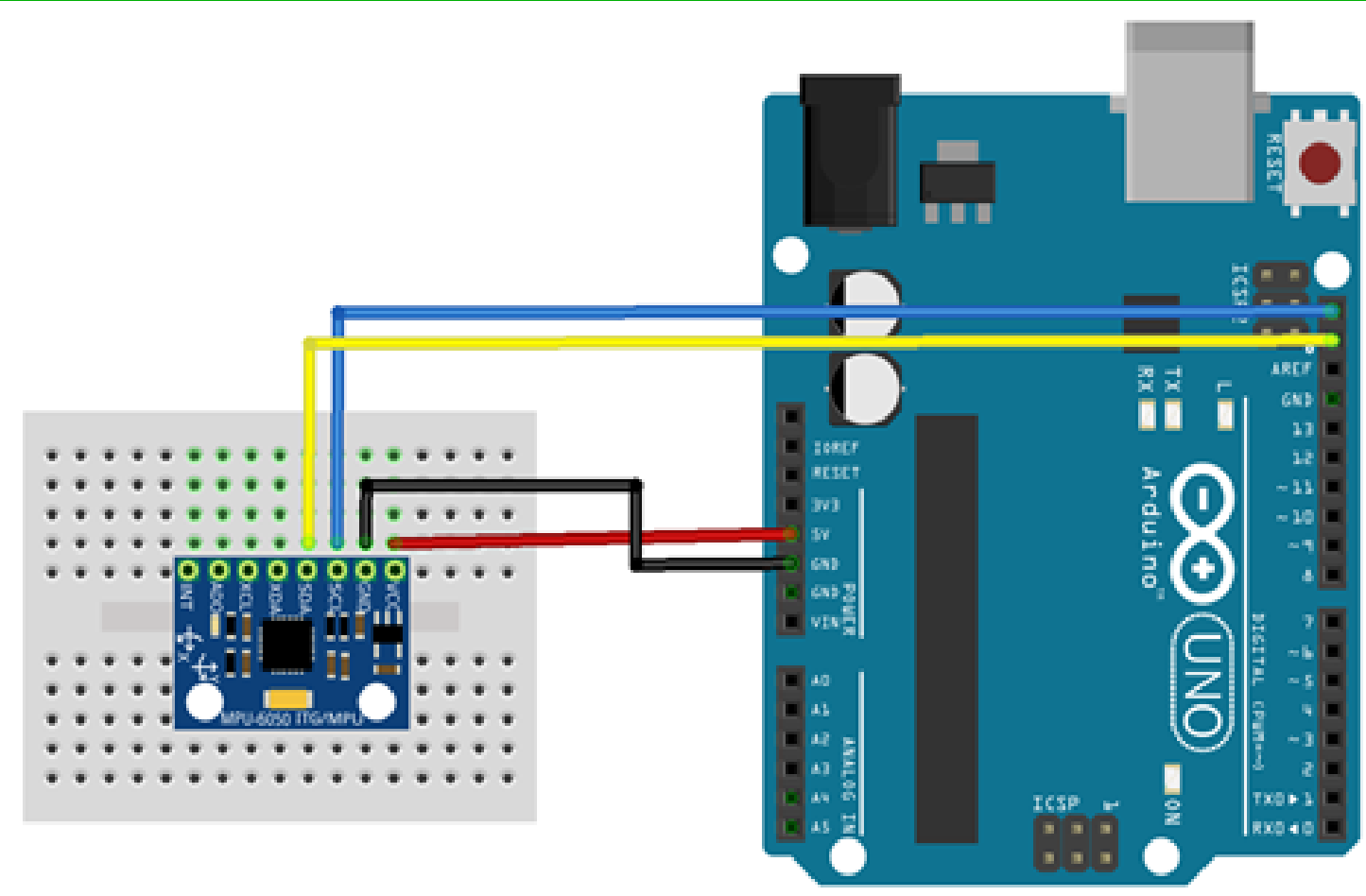

Рис. 1. Схема подключенияGY-521 к ArduinoUno

Следующим шагом была разработана программа для микроконтроллера, которая выполняет чтение информации с модуля микромеханического гироскопа и акселерометра по протоколу I2C, и формирует посылку для передачи через интерфейс RS-232. Результатом работы программы микроконтроллера стала последовательность чисел, состоящая из двух групп: показания акселерометра и гироскопа. Показания акселерометра представляют проекции на координатные оси ускорения свободного падения и представляют первые три столбца в логе. Названные параметры являются первичными данными, получаемыми от акселерометра. Вторая группа состоит из трех чисел и определяет углы ориентации, которые представляют четвертый, пятый и шестой столбец в логе. Данные гироскопа являются результатом обработки выполняемой на микроконтроллере, так как микромеханический гироскоп измеряет угловую скорость. Измерение углов производится относительно стартового положения с учетом текущей мгновенной угловой скорости и пройденного времени. Пример лога передаваемых данных показан на рис. 2. 


$\begin{array}{cccccc}\mathrm{a}_{\mathrm{x}} & \mathrm{a} y & \mathrm{a}_{\mathrm{z}} & \psi & \theta & \gamma \\ 11032 & 10996 & 4948 & -189 & -5 & 62 \\ 10976 & 10784 & 5496 & -189 & -4 & 62 \\ 10952 & 10724 & 5240 & -189 & -4 & 62 \\ 11096 & 10924 & 5244 & -188 & -4 & 62 \\ 10916 & 11160 & 4992 & -187 & -4 & 62 \\ 10868 & 11020 & 4936 & -187 & -4 & 61 \\ 10700 & 11160 & 4832 & -187 & -4 & 61 \\ 10852 & 11168 & 4828 & -187 & -4 & 61 \\ 10796 & 11132 & 4844 & -187 & -4 & 61 \\ 10840 & 11268 & 4988 & -187 & -4 & 61 \\ 10708 & 11224 & 4764 & -186 & -4 & 61 \\ 10692 & 10620 & 4876 & -186 & -4 & 60 \\ 10764 & 11200 & 4712 & -185 & -4 & 60 \\ 10704 & 11388 & 4740 & -185 & -4 & 60 \\ 10652 & 11652 & 4356 & -185 & -4 & 60 \\ 10588 & 11576 & 4516 & -185 & -4 & 60 \\ 10508 & 11624 & 4428 & -185 & -4 & 60 \\ 10536 & 11620 & 4408 & -185 & -4 & 60 \\ 10636 & 11604 & 4260 & -185 & -4 & 60 \\ 10568 & 11748 & 4192 & -184 & -4 & 60\end{array}$

\section{Рис. 2. Данные отправляемые микроконтроллером}

Данные формируемые микроконтроллером, отражаются управляющей программой, устанавливаемой на компьютере, к которому подключен стенд. Назначение программы управления стендом заключается в создании графиков, на основе данных полученных от стенда. Разработка программы проводилась на языке $\mathrm{C}++\mathrm{c}$ использованием инструментов, представляемых платформой .NET. Преимуществом выбора перечисленных инструментов для разработки является то, что разработанная программа позволяет работать на большом количестве компьютеров. Интерфейс, разработанной программы, а также результат её работы показан на рис 3.

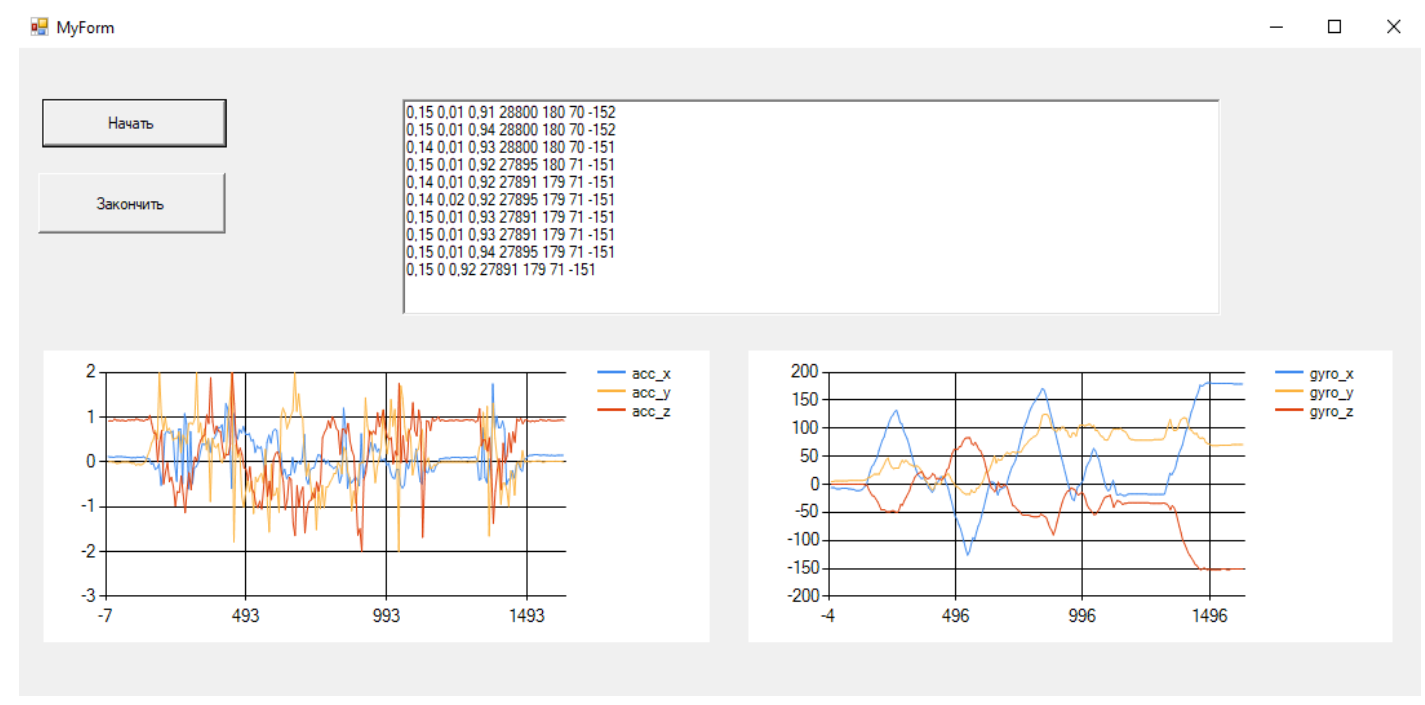

Рис. 3. Рабочее окно программы управления стенда 
На рис 3 представлена разработанная программа, элементами интерфейса являются: кнопки “Начать" и “Закончить", которые позволяют начать новое чтение данных от стенда и закончить соответственно; окно лога, содержащее числовые значения, получаемые от микроконтроллера; график показаний от акселерометра и график показаний гироскопа. Выполнение исследований работы микромеханических приборов требует обеспечения функций лога для накопления данных, получаемых от датчиков. Этот функционал реализуется автоматически, программа создает лог файл, в который записываются все данные получаемые от микроконтроллера.

Таким образом, был разработан лабораторный стенд, который позволяет изучать и исследовать работу микромеханических проборов: гироскопа и акселерометра, на примере выбранного модуля GY-521. Стенд состоит из микроконтроллера, подключаемого электронного модуля микромеханических приборов, и программы управления и отображения данных. При работе со стендом, пользователь, может в реальном масштабе времени исследовать реакции датчиков на различные виды перемещений и поворотов, накапливать необходимую информацию. Стенд может использоваться в других проектах, таких как, например, имитация поворота в пространстве подводного робота. Вместе с этим использованные элементы могут интегрироваться с системами автоматического управления, а разработанный стенд использоваться в виде подсистемы отражения информации контура обратной связи.

\section{Список литературы}

1. MPU-6000 and MPU-6050 Product Specification Revision 3.3 PS-MPU6000А-00. Руководство по эксплуатации. 2012 - 54 с.

2. Tutorial: How to use the GY-521 module (MPU-6050 breakout board) with the Arduino Uno//Michael Schoeffler[электронный ресурс]:-Режим доступа: https://mschoeffler.com/2017/10/05/tutorial-how-to-use-the-gy-521-module-mpu6050-breakout-board-with-the-arduino-uno/(12.11.2021). 